Original article

\title{
Nurses' perception of the ethical climate in the Iranian hospital environment
}

\author{
Vida Shafipour'1, Mahbobeh Yaghobian², Leila Shafipour ${ }^{3}$, Mohammad Reza Heidari $^{4 *}$
}

(Received: 6 Jun 2016; Accepted: 14 Apr 2016)

\begin{abstract}
Background and Purpose: Ethical climate is hinged upon organizational culture, rules, and policies; nurses' perception of this concept varies according to the ward they are working on and the new rules of the treatment system. This can exert adverse impact on their care method and performance. Thus, this study aimed to determine nurses' perceptions of the ethical climate governing the hospital environment.

Methods: This descriptive, cross-sectional study was conducted on 168 nurses working in a teaching hospital. The data were collected through census method, using a demographic form and Olson's Hospital Ethical Climate Survey (HECS). For data analysis, descriptive (percentage, frequency, mean, and standard deviation) and inferential statistics (independent t-test and ANOVA for the establishment of the relationship between ethical climate and demographic factors, as well as Friedman's test for ranking the factor) were used.

Results: Mean ethical climate scores of nurses for managers, hospital, physicians, patients, and colleagues factors were $3.87 \pm 0.71$, $3.39 \pm 0.68,3.00 \pm 0.67,3.68 \pm 0.57$, and $3.82 \pm 0.54$, respectively. The total mean score of ethical climate was $3.5 \pm 0.51$. The comparison among the mentioned factors indicated that managers $(P=0.000)$ factor acquired the highest score. In addition, organizational ethical climate did not show any significant association with gender, marital status, education level, working shift, and employment status, whereas there was a statistically significant relationship between job title and income $(P=0.000)$.

Conclusion: The highest score of ethical climate belonged to managers/ factor, while the minimum score was related to physicians. Regarding the role of ethical climate in the improvement of nurses' performance, planning for enhancing the ethical climate seems to be mandatory.
\end{abstract}

Keywords: Ethical climate, Hospital environment, Nurse

\section{Introduction}

Perception of ethical climate is an important aspect of organizational culture (1). For optimum performance and to oblige observance of rules and regulations, organizations need ethical principles to help with implementation of ethical behaviors and management practices $(2,3)$. In fact, the structure of organizational climate plays an important role in the organizational perceptions and behaviors, such as ethical climate (4).

Attention to the perception of organization's ethical climate increases sensitivity of managers to the improvement of staff compliance with ethical standards $(2,3)$. Ethical climate is one of the important factors in the formation of inter-organizational relationships $(5,6)$ and the consistency of the already-made decisions with ethical principles $(2,7)$, which influence the productivity and efficiency of the organization's performance (5). Nurses, as the largest labor force in health care systems, encounter different ethical decision-making problems in connection with

\footnotetext{
${ }^{1}$ Department of Medical-Surgical Nursing, Nasibeh Nursing \& Midwifery Faculty, Mazandaran University of Medical Sciences, Sari, Iran

${ }^{2}$ Department of Management Nursing, Nasibeh Nursing \& Midwifery Faculty, Mazandaran University of Medical Sciences, Sari, Iran

${ }^{3}$ Department of Emergency Medicine, Imam Khomeini Hospital, Mazandaran University of Medical Sciences, Sari, Iran

${ }^{4,}$ Corresponding author: Department of Nursing, School of Nursing \& Midwifery, Shahed University, Tehran, Iran. Email: heidari43@yahoo.com
} 
patients on a daily basis due to the availability of complex care situations (8-10). In fact, the ethical dimension should be taken into account in addition to the medical aspect in every decision that is made for patients (5).

Nurses' ability to process and resolve ethical issues is based upon the experience of ethical distress while interacting with patients and the experience of ethical problems arising from the ethical climate of the organization. For this reason, the quality of care delivery is influenced by the ethical climate $(8,11)$. Ethical climate addresses care-related problems as well as ethical issues. Perception of the ethical climate of work environments is based upon the relationship with colleagues, patients, managers, physicians, and hospital administrators in the face of ethical problems (12). This relationship is influenced by individuals' professional behavior, perception of which is necessary for the resolution of ethical problems (13).

With the adjustment of the factors related to ethical climate, it is possible to improve patient care and provide background for proper ethical decisionmakings $(2,14)$. Indeed, promotion of ethical climate leads to increased quality of care (15) and coping of nurses with other causes of dissatisfaction in the work environment (16).

In this regard, some studies suggested a significant positive relationship between nurses' level of moral distress in intensive care units (ICUs) and the ethical climate dominant over these units $(17,18)$. However, a study performed in Jordan has revealed participants' perception of the ethical climate of the hospital and its aspects cannot predict severity and frequency of moral distress among the nurses in ICUs (19). The ethical climate of workplace is an important factor in nurses' job satisfaction and efficiency, as well as in their decisions on whether to leave the position and even the profession or not $(15,16)$. Former studies showed that unsuitable ethical climate in the organization can play a critical role in creating moral distress, job dissatisfaction, and consequently, resignation from the nursing profession $(16,20-22)$.

Several factors, such as age, type of hospital, human resources, management approach, organizational philosophy, and values, affect nurses' perceptions of ethical climate $(6,14,23,24)$. The factors promoting organizational environment include positive ethical climate, fulfillment of patients' and their families' needs, and standard teamwork $(2,5,25)$, because there is a direct relationship between nurses' job satisfaction and the dimensions of ethical climate (23).

In previous studies, some ICU nurses had relatively favorable views on their work environment (26), whereas some others assessed their work environment at an average level (2).

The ethical climate of hospitals is key to creating a proper environment for the nursing care, and can be affected by various factors and working conditions, as well as management policies. In the same way, ethical climate varies from organization to organization and review of the related studies presents conflicting results regarding nurses' perceptions of the ethical climate dominant over their work environment. Thus, the present study was conducted to investigate perception of the nurses employed in a teaching hospital regarding the ethical climate in their work environment.

\section{Materials and Methods}

In this descriptive, cross-sectional study, 168 nurses were studied using a demographic form and Hospital Ethical Climate Scale (HECS), which was designed by Oslon in 1998 to measure nurses' perceptions of ethical climate dominant over their workplace or hospital based on Victor and Cullen's questionnaire. This scale evaluates nurses' perceptions of the relationship with colleagues, patients, managers, hospital, and physicians as well as their ability to cope with ethical issues in patient care $(12,14)$. This questionnaire includes 26 items and five factors, namely, patients, colleagues, managers, physicians, and hospital. The distribution of the items regarding the relationship of nurses with each of these factors is as follows: patients $(2,6,11,19)$, colleagues $(1,10$, $18,23)$, managers $(3,7,12,15,20,24)$, physicians $(5,9,14,17,22,26)$, and hospital $(4,8,13,16,21$, $25)$. The questionnaire was rated using a 5-point Likert scale ranging from almost never (1 point), seldom ( 2 points), sometimes ( 3 points), often (4 points), and almost always (5 points). 
The total score of the scale was obtained from calculating the sum of the item scores. The minimum and maximum scores for each item were 1 and 5 , respectively. Scores 1 and 5 indicate the lowest and highest agreement with the expression, respectively. Thus, the scoring continuum ranges from 26 to 130 . The higher the score, the more positive will be the ethical climate. In addition, 3.5 was considered the cut-off point in order to achieve a better overview and to make a better comparison of the scenarios. Indeed, any score above the cut-off point indicates the favorable opinion of the employees regarding the ethical climate and indicates positive climate $(27,28)$. The questionnaire was completed based on self-report technique. Validity of the scale was established by 12 experts and professionals. Khalesi et al. reported the Cronbach's alpha reliability coefficient of 0.94 for this scale (27). In the present study, its internal consistency was assessed and the Cronbach's alpha coefficient of 0.93 was obtained for the questionnaire. To analyze the data, descriptive (mean, percentage, and standard deviation) and inferential statistics, that is, independent t-test (for the relationship between ethical climate and demographics) and One-way ANOVA (for the association of education level and employment status with ethical climate) were used in SPSS, version 20.

Afterthe receiving of permission, the questionnaires were distributed among the participants. Before the distribution of the questionnaires, the researchers provided the necessary explanations for the nurses and received their verbal consent for participation in the study. Moreover, the nurses were assured of the confidentiality of the data.

\section{Results}

In this study, the questionnaires were distributed among all the nurses meeting the inclusion criteria and consented to participate in the study $(n=168)$. Mean age of the subjects was $35.31 \pm 6.06$ years (age range: 26 to 62 years). Mean duration of service was $10.3 \pm 5.05$ years (range: 1 to 28 years; Table 1 ).

In addition, the mean score of the nurses' perception
Table 1. Demographic characteristics of the nurses $(n=168)$

\begin{tabular}{lccc}
\hline Variable & & N & \% \\
\hline \multirow{2}{*}{ Gender } & Male & 35 & 20.8 \\
& Female & 133 & 79.2 \\
\hline \multirow{2}{*}{ Education level } & Associate's degree & 14 & 8.3 \\
& Bachelor's degree & 154 & 91.7 \\
\hline \multirow{2}{*}{ Marital status } & Married & 144 & 85.7 \\
& Single & 24 & 14.3 \\
\hline \multirow{3}{*}{ Employment status } & Official & 133 & 79.2 \\
& Trial & 5 & 3 \\
& Contractual & 20 & 11.9 \\
Job title & Agreement & 10 & 6 \\
\hline
\end{tabular}

of the ethical climate governing the hospital was $3.53 \pm 0.51$. The highest mean score belonged to the managers factor followed by colleagues, patients, hospital, and physicians, respectively (Table 2).

Comparison of the ranks among the factors, managers, and physicians obtained the highest and lowest ranks, respectively. Friedman's test also showed a significant relationship in this regard $\left(P=0.000, \mathrm{df}=4, \mathrm{X}^{2}=246.222\right)$.

The comparison of the mean of nurses' responses to HECS items showed that the highest score belonged to the item "My manager is someone I can respect" obtained from the managers factor. On the other hand, the lowest score pertained to the item "Physicians ask nurses for their opinions about treatment decisions" from the physicians factor (Table 3 ).

The normality of the data was assessed and confirmed by Kolmogorov-Smirnov test $(P=0.721)$. One-way ANOVA showed that organizational ethical climate was not significantly correlated with education level $(P=0.177, \mathrm{~F}=1.83)$, shift $(P=0.421, \quad \mathrm{~F}=0.978), \quad$ and employment status

Table 2. Mean score of ethical climate factors

\begin{tabular}{ll}
\hline Factor & Mean \pm SD \\
\hline Managers & $3.87 \pm 0.71$ \\
Hospital & $3.39 \pm 0.68$ \\
Physicians & $3.00 \pm 0.67$ \\
Patients & $3.68 \pm 0.57$ \\
Colleagues & $3.82 \pm 0.54$ \\
\hline
\end{tabular}


Table 3. Mean score of nurses' responses to hospital ethical climate survey items

\begin{tabular}{|c|c|c|}
\hline Factor & Item & $\operatorname{Mean} \pm$ SD \\
\hline \multirow{4}{*}{ Colleagues } & 1. My colleagues listen to my concerns about patient care & $3.75 \pm 0.71$ \\
\hline & 10. My colleagues help me with difficult patient care issues & $3.82 \pm 0.83$ \\
\hline & 18. I work with competent colleagues & $3.82 \pm 0.78$ \\
\hline & 23. Safe patient care is provided in my unit & $3.89 \pm 0.88$ \\
\hline \multirow{4}{*}{ Patients } & 2. Patients know what to expect from their care givers & $3.32 \pm 0.81$ \\
\hline & 6. Nurses have access to the information necessary to solve a patient care problem & $3.63 \pm 0.76$ \\
\hline & 11. Nurses use the information necessary to solve a patient care issue/problem & $3.74 \pm 0.86$ \\
\hline & 19. The patient's wishes are respected & $4.04 \pm 0.88$ \\
\hline \multirow{6}{*}{ Managers } & 3. When I am unable to decide what is right or wrong in a patient care situation, my manager helps me & $3.73 \pm 0.98$ \\
\hline & 7. My manager supports me in my decisions about patient care & $3.69 \pm 0.96$ \\
\hline & 12. My manager listens to me about patient care issues & $4.01 \pm 0.88$ \\
\hline & 15. My manager is someone I can trust & $3.95 \pm 0.95$ \\
\hline & 20. When my colleagues are unable to decide what is right or wrong in a patient care situation, I have observed that my manager helps them & $3.57 \pm 0.91$ \\
\hline & 24. My manager is someone I can respect & $4.28 \pm .84$ \\
\hline \multirow{6}{*}{ Hospital } & 4. Hospital policies help me with difficult patient care issues & $3.20 \pm 0.95$ \\
\hline & 8. A clear sense of the hospital's mission is shared among nurses & $3.44 \pm 1.03$ \\
\hline & 25. I am able to practice nursing in my unit as I believe it should be practiced & $3.78 \pm 0.87$ \\
\hline & 13. The feelings and values of all parties involved in a patient care issue/problem are taken into account when choosing a course of action & $3.16 \pm 0.97$ \\
\hline & 16. Conflict is openly dealt with, not avoided & $3.56 \pm 0.87$ \\
\hline & 21. There is a sense of questioning, learning, and seeking creative responses to patient care problems & $3.52 \pm 0.86$ \\
\hline \multirow{6}{*}{ Physicians } & 5. Nurses and physicians trust one another & $3.09 \pm 0.99$ \\
\hline & 26. Nurses are supported and respected in this hospital & $2.71 \pm 1.06$ \\
\hline & 9. Physicians ask nurses for their opinions about treatment decisions & $2.41 \pm 1.11$ \\
\hline & 14. I participate in treatment decisions for my patients & $3.20 \pm 0.90$ \\
\hline & 17. Nurses and physicians respect each other's opinions & $3.13 \pm 0.95$ \\
\hline & 22. Nurses and physicians respect one another & $3.44 \pm 0.96$ \\
\hline
\end{tabular}

$(P=0.243, \mathrm{~F}=1.4)$; however, it was shown that it is significantly correlated with job title $(P=0.000$, $\mathrm{F}=16.8)$ and income $(P=0.035, \mathrm{~F}=2.64)$. Moreover, independent t-test did not reveal any statistically significant difference between ethical climate and gender, as well as marital status. Pearson productmoment correlation reflected that ethical climate is not significantly correlated with age $(P=0.171$, $\mathrm{r}=0.106)$ and work experience $(P=0.109, \mathrm{r}=0.124)$.

\section{Discussion}

The results of this study indicated that from the perspective of the nurses, the mean of the majority of the HECS items was equaled to 3.5 out of 5 . Therefore, it can be argued that the ethical climate of the hospital was a par. Studies performed on this issue in Iran showed that the mean value of ethical climate was 3.166 out of 5 and nurses reported the ethical climate to be at a relatively desirable level $(2,29)$. A study conducted in America also reported the mean value of 3.23 out of 5 for ethical climate, where this value was regarded as average and above with no positive effect on ethical climate (17).

It seems that the cultural context of each area affects the ethical climate of its organizations. In addition, the staff in different hospital wards had different perceptions of the ethical climate.

In this relation, Ajoodaniyan et al. stated that the nurses working in ICU deemed the quality of the dominant ethical climate to be at an average level (30). In terms of ICU nurses' perceptions of ethical 
climate, a study found support for the negative relationship of moral distress and work pressure with ethical climate, such that ICU nurses expressed a better perception of ethical climate as a result of reduced work pressure (31). It should be considered that the individuals' perceptions and experiences of ethical climate are affected by organizational rules and procedures that vary due to the differences in individual circumstances and working systems.

The results of this study demonstrated that managers and physicians obtained the highest and lowest scores from among the five factors of HECS. The remaining scores were respectively attributed to colleagues, patients, and hospital. In this regard, several studies have reported the managers factor as the most desirable one and the physicians factor as the most undesirable one $(6,21)$. Nevertheless, in the study conducted by Fogel et al., the highest and lowest scores belonged to the colleagues and hospital factors, respectively (31). Jalali et al. also found support for this finding. In that study, the highest score of ethical climate belonged to the managers factor and the lowest score pertained to both patients and physicians factors from the perspective of nurses (2).

However, Pauly et al. reported the highest and lowest mean scores for the colleagues and hospital factors, respectively, followed by patients, managers, and physicians factors, respectively (15). In a study conducted by Mobasher et al. (2008), the managers factor received the highest mean score and the hospital and physicians factors obtained the lowest mean scores from the viewpoints of nurses (3). The results of these studies suggest that mutual cooperation and relations between nurses and physicians are at the lowest level from the perspective of nurses. Considering the importance of the nursing profession, this drawback should be dealt with as it might lead to the loss of mutual trust and respect, and ultimately, the quality of health care will experience a considerable decline.

Nonetheless, our findings were reflective of a cooperative climate among nurses and managers. It is noteworthy that the term "managers" here refers to the executives directly responsible for the Department of Nursing, such as matrons, supervisors, and head nurses rather than hospital administrators. On the other hand, it seems that the considerable impact of organizational culture on nurses makes it necessary to reconsider some beliefs, cultural norms, and ethical rules and regulations of organizations.

Furthermore, lower score of the physicians factor may also be due to the absence of shared care goals in the care team, which reflects the need for the improvement of inter-group relationships. From among the 26 items of the HECS, the item "My manager is someone I respect" received the highest mean score, while the item "Physicians ask nurses for their opinions about treatment decisions" acquired the lowest mean score. However, Jalali, et al. obtained the highest mean score for the item "My manager is someone I can trust" and the lowest mean score for the item "When I'm unable to decide what is right or wrong in a patient care situation, my manager helps me" (2). Khazani et al. also reported that the highest mean score belonged to the item "My manager is someone I respect" (29).

In some other studies, the two items of "My manager is someone I respect" and "When I am unable to decide what is right or wrong in a patient care situation, my manager helps me" received the highest mean scores. On the other hand, the two items of "Physicians ask nurses for their opinions about treatment decisions" and "Nurses are supported and respected in this hospital" obtained the lowest mean scores in those studies $(3,29)$. In Fogel's study (2007), nurses assigned the highest and lowest scores among the statements of HECS to the items "My peers help me with difficult patient care issues/problems" and "Conflict is openly dealt with, not avoided", respectively (31). Various studies have obtained different results regarding nurses' perceptions of ethical climate in the organization they are employed in; thus, it seems that cultural and management differences can influence some inter-firm relationships between nurses and their colleagues and/or authorities. Some effective factors in the ethical climate of hospital are unknown, which is one of the limitations of this study. It seems that this issue can be investigated via qualitative studies, as well.

\section{Conclusion}

The present study draws upon the ethical climate dominant over nurses' working environment. It 
was noted that nurses and managers had a desirable relationship, while nurses' relationship with physicians was at the lowest level. The ethical climate of hospitals is typical of the organizational atmosphere comprising of the interpersonal relations among the health care personnel as well as their relationships with patients and their families. Given the importance of ethical climate in hospitals for the creation of a suitable environment for professional performance and the type of nursing care provided for patients, managers are recommended to boost the spirit of cooperation and interaction among treatment groups by making the necessary plans. In addition, inappropriate ethical climate can lead to moral distress, job dissatisfaction, lack of accountability, low performance, and resignation from the nursing profession. Therefore, the authorities should take the necessary measures in parallel with nurses' perceptions and views to improve organizational atmosphere.

\section{Conflicts of interest}

None declared.

\section{Authors' contributions}

V. SH. and MR. H. designed the study, provided the important suggestions for the improvement of the first draft. VSH revised the paper, supervised the study process. L. SH. and M. Y. collected the data. MR. H. and V. SH. performed the statistical analysis and contributed to the study design. All the authors read and approved the manuscript.

\section{Acknowledgements}

The authors would like thank all the nurses who participated in this study.

\section{References}

1. Joolaee S, Jalili H, Rafiee F, Haggani H. The relationship between nurses' perception of moral distress and ethical environment in Tehran University of Medical Sciences. J Med Ethics Hist Med. 2011; 4(4):56-66. [Persion].

2. Jalali T, Kalantari S, Hekmat Afshar M, M jouybari L. The nurse's perception from the hospital ethical. Jentashapir. 2013; 4(1):65-72. [Persion].
3. Mobasher M, Nkhaee N, Garoosi S. Assessing the ethical climate of Kerman teaching hospitals . ijme. 2008; 1 (1) :45-52. [Persion].

4. Borhani F, Jalali T, Abbaszadeh A, Haghdoost AA, Amiresmaili M. Nurses' perception of ethical climate and job satisfaction.J Med Ethics Hist Med. 2012; 5(6):1-6.

5. Cullen JB, Parboteeah KP, Vtictor B. The effects of ethical climates on organizational commitment: A two-study analysis. J Bus Ethics 2003; 46(2):127-41.

6. Borhani $\mathrm{F}$, Jalali $\mathrm{T}$,Dehestani $\mathrm{M}$, Abbaszadeh A, Torabi J, Esmaeilpour H . Ethical climate in social security and private hospitals in Kerman in 2012. Journal of Bioethics 2013;4(14): 67-81.

7. Montakab Yegane M, Arshadi N, Baharlo M, Changi Ahmadi S. The relationship between organizational climate and moral, leader-member exchange And perceived external prestige on organizational commitment. Journal of Social Psychology 2013; 8(29):61-73. [Persion].

8. Schluter J, Winch S, Holzhauser K, Henderson A. Nurses' moral sensitivity and hospital ethical climate: a literature review. Nurs Ethics. 2008 May;15(3):304-21.

9. Baldwin K. Moral distress and ethical decision making. Nursing Made Incredibly Easy 2010; 8(6):5.

10. Claeys M, Faelens A, Sabbe B, Schrijvers D, Dierckx de Casterle B, Luyten P. Psychometric properties of the Hospital Ethical Climate Survey: a cross-sectional study in a large sample of Belgian psychiatric nurses. European Journal for Person Centered Healthcare 2012; 1 ( 1) : 202-208.

11. Ulrich CM, Taylor C, Soeken K, O’Donnell P, Farrar A, Danis M, Grady C. Everyday ethics: ethical issues and stress in nursing practice. J Adv Nurs. 2010 Nov;66(11):2510-9.

12. Olson L. Ethical climate in health care organizations. Int Nurs Rev. 1995; 42(3):85-90.

13. Shakerinia I. The ethical climate relationships with organizational supports in organizational behaviors of nurses in public hospitals in Rasht province. Hospital, 2012 11(1).28-19. [Persion].

14. Barnett T, Vaicys Ch.The moderating effect of individuals' perceptions of ethical work climate on ethical judgments and behavioral intentions. Journal of Business Ethics 2000. 27(4): 351-362.

15. Pauly B, Varcoe C, Storch J, Newton L. Registered nurses' perceptions of moral distress and ethical climate. Nurs Ethics. 2009; 16(5):561-73.

16. Joolaee S, Jalili HR, Rafii F, Hajibabaee F, Haghani H. The relationship between ethical climate at work and job satisfaction among nurses in Tehran .Indian J Med Ethics. 2013; 10(4):238-42.

17. Corley MC ,Minick P, Elswick RK, Jacobs M. Nurse moral distress and ethical work environment. Nurs Ethics. 2005; 12(4):381-90.

18. Ulrich C, O’Donnell P, Taylor C, Farrar A, Danis M, Grady C. Ethical climate, ethics stress, and the job satisfaction of 
nurses and social workers in the United States. Soc Sci Med. 2007; 65(8):1708-19.

19. Allari R, Abu-Moghli F. Predictors of moral distress among jordanian critical care nurses. International Journal of Nursing Science 2013, 3(2): 45-50.

20. Hart SE. Hospital ethical climates and registered nurses' turnover intentions. Image J Nurs Scholarsh 2005; 37: 173-77.

21. Borhani F, Hoseini SH , Abbaszadeh A, Abbasi M, Fazljoo E. Nurses' perception of ethical climate governing the teaching hospital affiliated with the the university of Medical Sciences Shahid Sadughi Yazd..J Med Ethics. 2014; 8(29):41-65.

22. Hwang JI, Park HA. Nurses' perception of ethical climate, medical error experience and intent-to-leave. Nurs Ethics. 2014; 21(1):28-42.

23. Goldman A, Tabak N. Perception of ethical climate and its relationship to nurses' demographic characteristics and job satisfaction. Nurs Ethics. 2010; 17(2):233-46.

24. García IG, Castillo RF, Santa-Bárbara ES. Nursing organizational climates in public and private hospitals.Nurs Ethics. 2013 11; 21(4):437-446.

25. Silén M, Svantesson M, Kjellström S, Sidenvall B, Christensson L. Moral distress and ethical climate in a Swedish nursing context: perceptions and instrument usability. J ClinNurs. 2011; 20(23-24):3483-93.
26. Ajoodaniyan N, sorushan V, rahmani A, Ghaderi B. Ethical climate governed on hospital environments from the perspective of nurses employed in intensive care units. J Med Ethics Hist Med; 2010; 4(13): 39-59.

27. Khalesi N, Arabloo J, Khosravizadeh O, Taghizadeh S, Heyrani A, Ebrahimian A. Psychometric properties of the Persian version of the "Hospital Ethical Climate Survey”. J Med Ethics Hist Med, 2014; 7(15):1-7.

28. Suhonen R, Stolt M, Gustafsson ML, Katajisto J, Charalambous A. The associations among the ethical climate, the professional practice environment and individualized care in care settings for older people. J Adv Nurs. 2014 Jun;70(6):1356-68.

29. Khazani S, Shayestehfard M, Saeed-al-Zakererin M, Cheraghian B. Nurses' perception of actual and ideal organizational ethical climate in hospitals of Ahwaz Jondishapour University of Medical Sciences. ijme 2013;2(6):99-110.

30. Ajoodaniyan N, sorushan V, rahmani A, Ghaderi B. Ethical climate governed on hospital environments from the perspective of nurses employed in intensive care units. J Med Ethics Hist Med; 2010; 4(13): 39-59.

31. Fogel, KM. The relationship of moral distress, ethical climate, and intent to turnover among critical care nurse. Ph.D Dissertation in philosophy. University of Chicago. 2007. 